\title{
DNA Translocation through Low-Noise Glass Nanopores
}

\author{
Lorenz J. Steinbock, Roman D. Bulushev, Swati Krishnan, Camille Raillon, and Aleksandra Radenovic* \\ Laboratory of Nanoscale Biology, Institute of Bioengineering, School of Engineering, École Polytechnique Fédérale de Lausanne, 1015 Lausanne, Switzerland. \\ The manuscript was written by L. Steinbock. Experiments were planned by L. Steinbock and A. Radenovic. Experiments were conducted by L. Steinbock, R. Bulushev, \\ and S. Krishnan. C. Raillon drilled the membrane nanopores. Resources were provided by A. Radenovic.
}

\begin{abstract}
The effect of electron irradiation-induced shrinking on glass nanocapillaries with diameters ranging from 75 to $14 \mathrm{~nm}$ was analyzed by measuring the conductance characteristics with and without DNA translocation. We have investigated nanocapillary shrinking with a scanning electron microscope from several perspectives to understand the geometry of the shrunken nanocapillary. On the basis of this observation, the conductance was modeled with respect to the nanocapillary diameter, which allowed reproducing the experimental results. We then translocated DNA through the shrunken nanocapillaries and measured higher
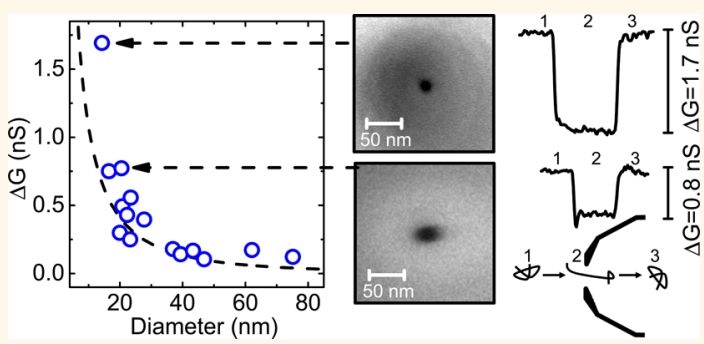
conductance drops for smaller diameters, reaching $1.7 \mathrm{nS}$ for the $14 \mathrm{~nm}$ diameter nanocapillary. A model taking into account the conical shape of the shrunken nanocapillaries also supported this dependence. Next, we calculated the noise in the form of the standard deviation of the ionic conductance (between 0.04 and $0.15 \mathrm{nS}$ ) to calculate a signal-to-noise ratio (SNR) and compared it with nanopores embedded in $20 \mathrm{~nm}$ thick silicon nitride membranes. This shows that although nanocapillaries have smaller signal amplitudes due to their conical shape, they benefit from a lower noise. The glass nanocapillaries have a good SNR of about 25 compared with the SNR of 15 for smaller sized nanopores in silicon nitride membranes. The ability to use a modified model of nanopores to mimic the block conductance by DNA translocation provides a theoretical framework to support experimental results from translocating polymers such as DNA.
\end{abstract}

KEYWORDS: nanopore $\cdot$ nanocapillary $\cdot$ quartz glass $\cdot$ DNA translocation $\cdot$ resistive pulse technique $\cdot$ scanning electron microscope

S olid-state nanopores for single-molecule detection of DNA were first used in 2001 by Li et al., who were able to detect double-stranded (ds) DNA when it was translocated through the nanopore. ${ }^{1}$ To fabricate this nanopore, an electron beam from a transmission electron microscope (TEM) was focused on a few nanometer thick silicon nitride membrane. The membrane separates two reservoirs containing a salt solution, across which an electric potential was applied. This causes an ionic current that is measured and depends on parameters such as salt concentration and the dimension of the nanopore. Nanopores are a useful technique to detect charged single molecules, which can be forced to translocate through the nanopore by the electric field. The volume displaced by the molecule can be sensed, because when it enters the nanopore, there is a reduction in the ionic current at high salt concentrations. $^{2}$

The ability to precisely control the size of the nanopore is crucial to sense small molecules since the amplitude of the conductance drop increases with smaller diameters. An early technique used the electron beam of an already drilled nanopore to shrink it to any desired diameter. ${ }^{3}$ The electron beam would heat up the silicon membrane around the nanopore, reducing the size of the pore due to the surface stress and the increased mobility of the heated atoms. The method of pore shrinking with a transmission electron microscope was soon expanded to other instruments such as scanning electron microscopes or lasers, which were able to locally heat up a material. ${ }^{4-6}$ An opposite approach was reported by Beamish et al. by enlarging solidstate nanopores using a high electric field. ${ }^{7}$

An inexpensive alternative to solid-state nanopores in silicon membranes is laser pulled glass nanocapillaries, which have a conical shape and a very small orifice at their tip. ${ }^{8}$ It was shown that they are able to sense the folding state of translocating dsDNA on the single-molecule level. ${ }^{9}$
* Address correspondence to aleksandra.radenovic@epfl.ch.

Received for review September 26, 2013 and accepted November 13, 2013.

Published online November 26, 2013 10.1021/nn405029j

C 2013 American Chemical Society 
Moreover, nanocapillaries are user-friendly substitutes for nanopores in combination with optical tweezers, profiting from a high force resolution and force application in the XY-plane. ${ }^{10-13}$ Recently nanocapillaries have found various applications and enhancements through lipid coatings or by serving as scaffold apertures for DNA origami nanopores. ${ }^{14,15}$ The possibility to image and shrink nanocapillaries without the need to coat the glass with a conducting layer has expanded the capabilities of this new source of nanopores. ${ }^{16}$ Small diameters have the ability to increase the signalto-noise ratio because they cause higher signal amplitudes for translocating DNA. This has important consequences since it allows for detecting and differentiating smaller molecules, which is especially important in DNA sequencing or protein detection. ${ }^{17,18}$ The relationship between the pore geometry and the amplitude of the current decrease was investigated in various theoretical and experimental conditions. ${ }^{19-21}$ While Wanunu et al. underlined the importance of using a thin membrane to generate short nanopores for an amplitude decrease, Kowalczyk et al. highlighted the benefits of a small nanopore to increase the signal amplitude of translocating DNA. ${ }^{22,23}$

This work explores the newly developed technique of shrinking nanocapillaries to diameters ranging from 100 to $10 \mathrm{~nm}$ and evaluates the effect of smaller diameter on the current decrease caused by a single dsDNA molecule. The shrinking capability allows for the use of nanocapillaries with bigger orifices and shorter tapers and also leads to a second steeper cone. These two shape factors cause the nanocapillaries to resemble more classical nanopores in membranes and to profit from their higher signal amplitudes. This can be demonstrated with small-diameter nanocapillaries reaching conductance drops of $1.7 \mathrm{nS}$ for one dsDNA molecule. Further, a model was established to reproduce the conductance and the conductance drop due to DNA translocation as a function of the diameter of conical glass nanopores. The fit of the model to the experimental data is in good qualitative and quantitative agreement and supports the validity of the theoretical assumptions. By calculating the standard deviation of the conductance traces we are able to quantify the ionic conductance noise and to deduce the SNR. The SNR of the shrunken glass nanopores was compared with the similar sized nanopores in silicon nitride membranes. Glass nanopores possess a higher SNR of about 25 for a $14 \mathrm{~nm}$ diameter orifice, while a silicon nitride nanopore of about $3 \mathrm{~nm}$ diameter has a SNR of about 15. This illustrates that besides strategies that focus on the production of small and thin nanopores for high signal-to-noise performances, one has to also focus on the noise behavior of the nanopore. Future nanopore research could therefore test new membrane materials or decrease the area of the membrane in order to lower the capacitance and improve the noise in the ionic current. ${ }^{24,25}$ One avenue would be the development of low-noise nanopores embedded in very thin glass layers. ${ }^{26}$

\section{RESULTS AND DISCUSSION}

Previous findings gave us the capability to image and shrink glass nanocapillaries to any size between approximately 10 and $200 \mathrm{~nm} .^{16}$ This allows us to investigate the effect of different sized glass nanopores on the blocked conductance caused by a single dsDNA molecule. Only nanocapillaries shrunken to diameters below $80 \mathrm{~nm}$ were used for the DNA translocation experiment. The shrinking process decreased the taper length and increased the glass thickness in addition to reducing the diameter of the nanopore at the tip (see scheme in Figure 1a). Therefore, by analogy to nanopores one would expect overall benefits in terms of an increased SNR for a shrunken nanocapillary. ${ }^{22,23}$ A microscopy image of the whole nanocapillary can be seen in Figure 1b, which illustrates the conical shape. To visualize the shrinking and its effect on the shape, a side-view image was taken with the scanning electron microscope (SEM) (see Figure 1c). At its very end the nanocapillary has a width of about $230 \mathrm{~nm}$ (see black arrow in Figure 1c). When imaged from the top, the nanocapillary revealed a nanopore with a diameter of around $190 \mathrm{~nm}$ (see Figure 1d).

The width of $230 \mathrm{~nm}$ of the nanocapillary and the $190 \mathrm{~nm}$ orifice reveal a glass thickness of about $20 \mathrm{~nm}$. After 2 min of continuous imaging at $2 \mathrm{kV}$ and $529 \mathrm{pA}$ and having a working distance of $3.7 \mathrm{~mm}$, the diameter decreased to $27 \mathrm{~nm}$ (see Figure 1e). A bigger ring (indicated by white arrows in Figure 1e) of about $175 \mathrm{~nm}$ can be observed around the black nanopore. To illustrate the effect on the irradiated area, a second side view was taken of the nanocapillary (see Figure $1 \mathrm{f}$ ). To better compare it with the original shape of the nanocapillary, the contour of the nanocapillary in Figure 1c was overlaid as a black line. Care was taken to perform a proper overlay by using reference points such as the white dot at the bottom of the nanocapillaries of Figure 1c and $\mathrm{f}$. The shrunken nanocapillary has significantly decreased in its width at the tip from $230 \mathrm{~nm}$ to $180 \mathrm{~nm}$ (indicated by two white arrows in Figure 1f). This value corresponds well with the diameter of $175 \mathrm{~nm}$ from the previously described second ring in Figure 1e (white arrows in Figure 1e), revealing a thickening of the glass wall from around $25 \mathrm{~nm}$ to about $75 \mathrm{~nm}$. Further, a clear shortening of about $360 \mathrm{~nm}$ is observed when comparing the new tip end and the previous tip end from the imposed contour shape (see black arrow in Figure 1f). From this analysis a qualitative illustration of the shrinking effect is depicted in Figure 1a. The dashed line represents the nanocapillary before shrinking, and the continuous line represents the nanocapillary after shrinking. The scheme demonstrates the main effects of the 

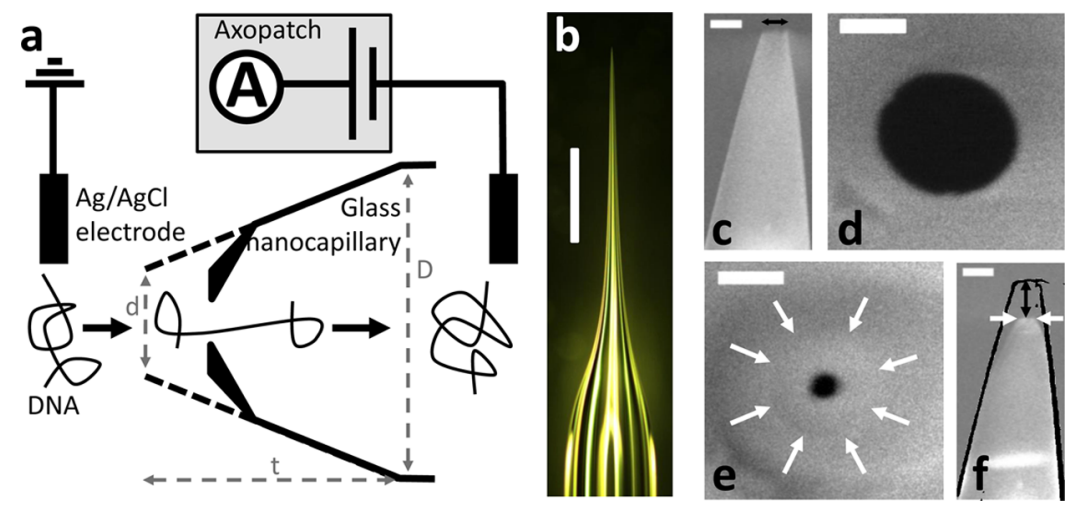

Figure 1. (a) Schematic of the setup. The Axopatch allows the application of a potential and the measurement of the ionic current. Two Ag/AgCl electrodes are placed on both sides of the nanocapillary. Addition of the DNA to the left cis reservoir and application of a positive potential to the right trans electrode causes the DNA to translocate through the glass nanopore. (b) Dark field image of a typical pulled nanocapillary whose cone has a taper length of approximately $2 \mathrm{~mm}$ from the tip to the shaft. The scale represents $0.5 \mathrm{~mm}$. (c) Side view of the tip of a nanocapillary taken with the SEM before shrinking. The black arrows highlight the $230 \mathrm{~nm}$ broad tip. The scale bar is $300 \mathrm{~nm}$. (d) Top view of the nanocapillary showing the nanopore with a diameter of $190 \mathrm{~nm}$ before shrinking with the SEM. The scale bar is $100 \mathrm{~nm}$. (e) After continuously imaging the nanocapillary for $2 \mathrm{~min}$ the inner diameter has shrunken to approximately $27 \mathrm{~nm}$. The white arrows depict the second ring with a diameter of $175 \mathrm{~nm}$, representing the thickness of the glass capillary. The scale bar is $100 \mathrm{~nm}$. (f) SEM side view of the nanocapillary after shrinking. The black line contours the profile of the original nanocapillary, while the black arrow illustrate the distance of $360 \mathrm{~nm}$ the capillary has shortened. The white arrows mark the shrunken tip, which is now only $180 \mathrm{~nm}$ wide. The scale bar is $300 \mathrm{~nm}$.

shrinking: decreasing of the nanopore diameter, thickening of the glass wall, and shortening of the taper length. Figure S1 in the Supporting Information (SI) contains a calculation of the volume of the two cones before and after shrinking. The two volumes differ by only $20 \%$, which is explained by the underestimation of the shrunken volume due to the bulged shrunken nanocapillary.

Next, 15 nanocapillaries were shrunken or left unmodified to investigate the relationship between the diameter and the conductance when filled with $1 \mathrm{M}$ $\mathrm{KCl}$. This allowed, for the first time, the establishment of the diameter-conductance relationship for conical glass nanocapillaries. Figure $2 \mathrm{a}$ shows a nanocapillary with a diameter of $237 \mathrm{~nm}$ that was shrunken to about $75 \mathrm{~nm}$ (see Figure 2b). An I-V curve was recorded, and the linear fit revealed a conductance of $217 \mathrm{nS}$ (see Figure 2c). As expected, when shrinking a nanocapillary to a diameter smaller than $14 \mathrm{~nm}$, the conductance was only $19 \mathrm{nS}$ (see Figure 2d, e, and f). To analyze this dependence, the conductance of nanocapillaries with various diameters was measured. Figure $2 \mathrm{~g}$ presents the conductance of nonshrunken capillaries (black circles) and shrunken nanocapillaries (hollow blue circles).

To understand the relationship between the conductance and the diameter of the nonshrunken capillaries, we determined the length of the taper from 40 optical micrograph images and plotted them in a cumulative count graph (see Figure S2). The black, filled circles were fitted with the following equation describing the conductance $(G)$ of a cone: ${ }^{21-23}$

$$
G=\sigma\left[\frac{4 t}{\pi d D}+\frac{1}{2 d}+\frac{1}{2 D}\right]^{-1}
$$

where $D$ is the diameter of the nanocapillary at the shaft $(0.3 \mathrm{~mm}), d$ the diameter of the nanopore at the tip, $\sigma$ the specific conductance of the salt solution $(10.5 \mathrm{~S} / \mathrm{m}$ at $1 \mathrm{M} \mathrm{KCl}$ ), and $t$ the taper length (see Figure $1 \mathrm{~A}$ ). Fitting eq 1 to the experimental data (see continuous line in Figure $2 \mathrm{~g}$ ) resulted in a value of 3.3 $\mathrm{mm}$ for $t$, which is $50 \%$ greater than the experimental value determined in Figure S2 in the Supporting Information. Using the actual $t$ value of $2.1 \mathrm{~mm}$ resulted in the dashed line in Figure $2 \mathrm{~g}$. One reason for the greater $t$ value of $3.3 \mathrm{~mm}$ obtained by using the fitting parameter compared to the experimental value of $2.1 \mathrm{~mm}$ could lie in the semi-hourglass shape of the nanocapillary, which is not well represented by a simple cone. A more realistic approach is to simplify the nanocapillary as a double cone, with the first cone representing the longer and less steep tip of the nanocapillary and the second cone representing the steeper angle of the nanocapillary at the shaft (see SI Figure S3). Calculating the conductance of this double cone resulted in almost the same line as the fit (see continuous line in Figure 2g). This demonstrates that the hourglass shape is important and that a fit with a simplified model like that described by eq 1 results in an overestimation of the taper length $t$. Nevertheless, both models agree qualitatively with the overall linear trend between the diameter and the conductance.

Having established a good theory to model the conductance of micro- and nanocapillaries with diameters between 30000 and $100 \mathrm{~nm}$, we now focused on the conductance of nanocapillaries shrunken below $100 \mathrm{~nm}$ (see hollow, blue circles in Figure $2 \mathrm{~g}$ and $\mathrm{h}$ ). These nanocapillaries were all shrunken from diameters 


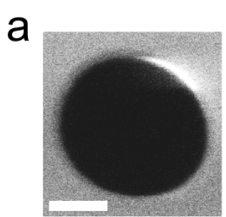

d

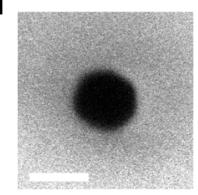

b

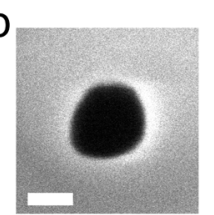

e

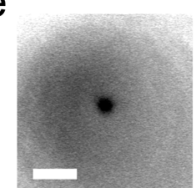

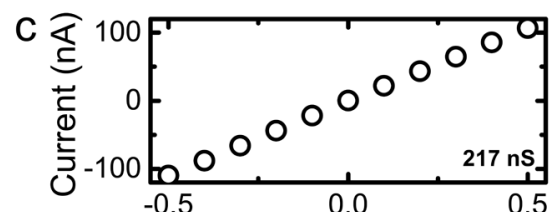

$f$
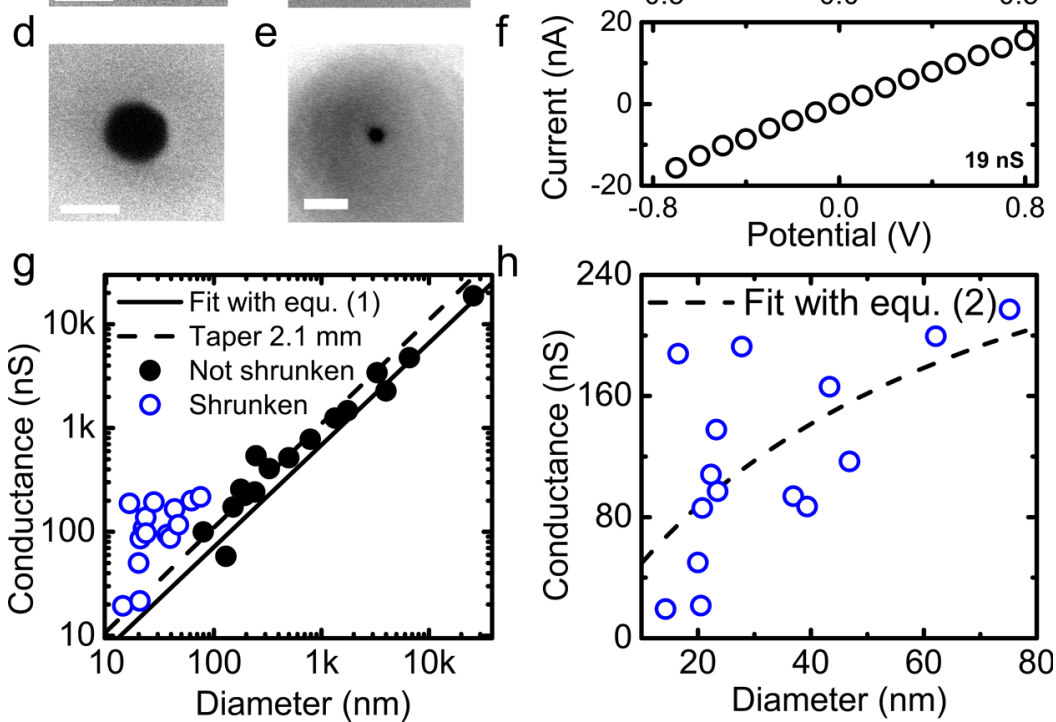

Figure 2. SEM image of the unmodified (a) and shrunken (b) nanocapillary. The left nanopore has a diameter of about $237 \mathrm{~nm}$. The scale bar is $100 \mathrm{~nm}$. (b) SEM image of the nanocapillary after shrinking to a nanopore diameter of approximately $75 \mathrm{~nm}$. The scale bar measures $50 \mathrm{~nm}$. (c) I-V curve of the respective nanocapillary in a $1 \mathrm{M} \mathrm{KCl}$ solution, which after a fit with a linear function reveals a conductance of $217 \mathrm{nS}$. ( $d$ and e) SEM images of a nanocapillary before and after shrinking with a diameter of about 91 and $14 \mathrm{~nm}$, respectively. Again the scale bars are 100 and $50 \mathrm{~nm}$, respectively. (f) $I-V$ curve of the shrunken nanocapillary in a $1 \mathrm{M} \mathrm{KCl}$ solution with a conductance of $19 \mathrm{nS}$. (g) Conductance as a function of the diameter of shrunken (hollow circles) and unmodified (filled circles) nanocapillaries. The continuous line is a fit with eq 1, leaving the taper length $t$ as the fitting parameter. The dashed line is the result of eq 1 using the actual taper length of $2.1 \mathrm{~mm}$. (h) Zoom of (g), focusing on the conductance dependence from the diameter of the shrunken nanocapillaries. The dashed line is a fit with eq 2.

ranging from approximately 100 to $200 \mathrm{~nm}$ as presented in Figure $2 a$ and d. Although fabricated with the same pull parameters, they have higher conductance values than the unmodified nanocapillaries and the corresponding fit. This is certainly due to the shrinking and the effect it has on the shape of the tip illustrated in Figure $1 \mathrm{c}$ to $\mathrm{f}$. Therefore eq 1 was modified to take into account the conductance of two cones as shown in Figure 1a: the conductance of the hourglass shape from the shaft to the nonshrunken part of the tip and the cone of the shrunken tip itself (see SI Figure S4a for scheme):

$$
G\left(d_{\mathrm{s}}\right)=\sigma\left(\left(\frac{4 t_{\mathrm{s}}}{\pi d_{\mathrm{s}} D_{\mathrm{s}}}+\frac{1}{2 d_{\mathrm{s}}}\right)+\left(\frac{4 t}{\pi D_{\mathrm{s}} D}+\frac{1}{2 D}\right)\right)^{-1}
$$

where $D$ is the diameter of $0.3 \mathrm{~mm}$ at the shaft, $D_{s}$ the diameter at the tip of the unmodified hourglass nanocapillary, $d_{s}$ the diameter of the nanopore at the tip of the shrunken nanocapillary, $t$ the taper length of the nonshrunken part, which was determined before to be 3.3 $\mathrm{mm}$, and $t_{\mathrm{s}}$ the taper length of the shrunken part of the nanocapillary. Equation 2 was fit to the conductance values of the shrunken nanocapillaries shown in Figure $2 \mathrm{~h}$ as a dashed line. Parameters $t_{\mathrm{s}}$ and $D_{\mathrm{s}}$ were the fitting values, which resulted in values of $543 \mathrm{~nm}$ for $t_{\mathrm{s}}$ and $514 \mathrm{~nm}$ for $D_{s}$. The obtained values are in good agreement with the observed shape of the shrunken nanocapillary in Figure $1 d$ to $f$ and the dimensions of the shrunken cone in SI Figure S4b.

Subsequently, $\lambda$-DNA was translocated through shrunken nanocapillaries that were immersed in $1 \mathrm{M}$ $\mathrm{KCl}$. From previous experiments, it is known that DNA translocation in these salt concentrations causes decreases in the ionic current. ${ }^{2,27}$ Two typical experiments can be seen in Figure $3 a$ and $b$. Figure $3 a$ presents the $\lambda$-DNA translocation through a $75 \mathrm{~nm}$ wide nanocapillary (see inset) at a potential of $0.3 \mathrm{~V}$. Characteristic step-like decreases in the centered conductance occurred (see Figure 3a). Eight typical events are shown, depicting the effect of the DNA folding state on the conductance decrease. The first two show only one level of blockade caused by the translocation of an unfolded DNA molecule. ${ }^{9,28}$ Here, at each time of the translocation, only one DNA molecule resides in the glass nanopore. The next two events are caused by partially folded DNA, indicated by a deeper decrease at the beginning caused by the presence of two dsDNA molecules inside the nanopore, followed by a lower decrease that is again caused by only one DNA strand. The following four events are either caused by two or more dsDNA molecules inside the constriction and are caused by folded DNA molecules. The histogram 


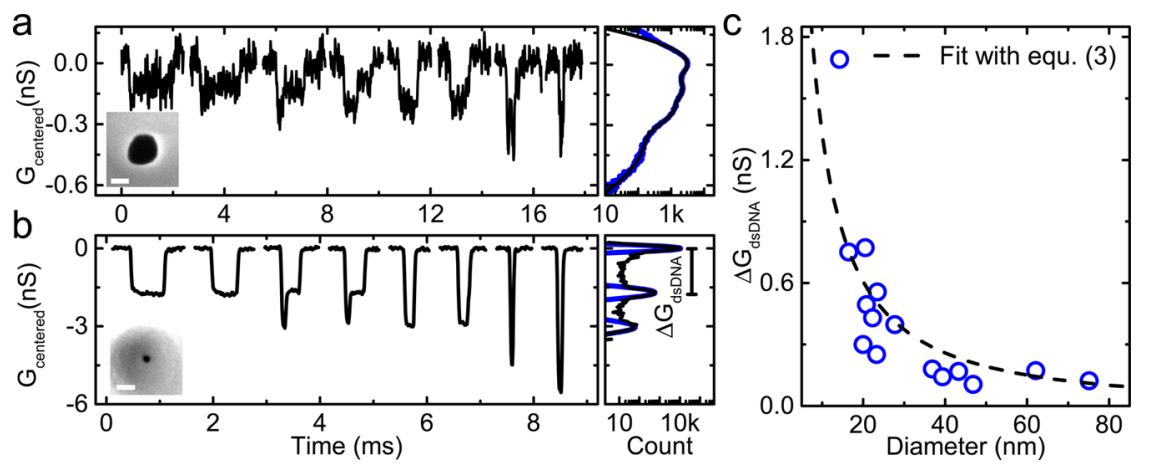

Figure 3. (a) Conductance traces were centered at $0 \mathrm{nS}$ by subtracting the mean baseline conductance. After addition of $\lambda$-DNA and application of $0.4 \mathrm{~V}$ to the trans electrode, typical translocation events are observed, revealing conductance reductions. The inset shows the respective nanocapillary with a diameter of $75 \mathrm{~nm}$. The corresponding histogram is quantized in peaks that are hard to distinguish due to the noise and the small amplitude. The continuous blue line is a fit with a multiple Gauss function. (b) $\lambda$-DNA translocation at a potential of $0.5 \mathrm{~V}$ through a $14 \mathrm{~nm}$ wide nanocapillary. Due to increased amplitude, the quantized peaks in the histogram can be easily distinguished. The distance between the top and the middle peak in the histogram represents the blocked conductance $\left(\Delta G_{\mathrm{dsDNA}}\right)$ by one dsDNA molecule and is determined by the multiple Gauss fit represented as a blue line. (c) Blocked conductance caused by one dsDNA molecule determined from the Gauss fits plotted as a function of the nanocapillary diameter. The translocation occurred at potentials between 0.3 and $0.6 \mathrm{~V}$. One can see an exponential increase with smaller diameters. The dashed line is a fit with eq 3 and shows good agreement with the experimental data.

contains the count of several hundreds of events (black line) and the multiple Gauss fit revealing the position of the steps at about 0.1 and $0.2 \mathrm{nS}$ (blue line). Noticeable is the high noise compared to the amplitude of the events, preventing an easy detection of the quantized steps. Figure $3 \mathrm{~b}$ demonstrates the translocation of $\lambda$-DNA through a $14 \mathrm{~nm}$ wide nanocapillary (see inset). As expected, the translocation events had much higher amplitudes, reaching about $1.7 \mathrm{nS}$ at $0.5 \mathrm{~V}$ (see Figure $3 b$ ). Besides the increase in the amplitude, the histogram on the right proves that the noise is relatively small, allowing a much easier identification of the peaks. SI Figure S5 contains the complete current trace recorded with this $14 \mathrm{~nm}$ wide nanopore, while $\mathrm{SI}$ Figure $\mathrm{S} 6$ explains the different DNA folding states of the translocating DNA.

Next we investigated the blocked conductance $\left(\Delta G_{\mathrm{dsDNA}}\right)$ caused by a single dsDNA molecule as a function of the respective diameter of the nanocapillary. $\Delta G_{\mathrm{dsDNA}}$ was calculated from the histogram shown in Figure $3 \mathrm{a}$ and $\mathrm{b}$ by calculating the distance between the top baseline peak and the second peak representing one dsDNA molecule in the nanocapillary. Figure $3 c$ presents the experimental amplitude values of 15 nanocapillaries with different diameters as hollow circles. To model the blocked conductance, a similar approach used by Kowalczyk et al. was used: ${ }^{23}$

$$
\begin{aligned}
\Delta G & =G_{\text {open pore }}-G_{\text {with DNA }} \\
& =G(d)-G\left(d_{\text {with DNA }}\right)
\end{aligned}
$$

$G_{\text {open pore }}$ is the open pore conductance plotted in Figure $2 \mathrm{~h}$ and described by eq $2 . G_{\text {with }}$ DNA is the conductance of a nanopore holding a single dsDNA molecule, $d_{\mathrm{DNA}}$ inside, which has a diameter of $2.2 \mathrm{~nm}$. The diameter $d_{\text {with DNA }}$ is described by $d_{\text {with DNA }}=\left(d^{2}-d_{\text {DNA }}^{2}\right)^{1 / 2}$. Fitting eq 3 to the experimental values displayed in
Figure $3 c$ with the length of the sensing taper as the fitting parameter permitted us to calculate the latter. The fit was able to reproduce the experimental data well and gave a value of $32 \mathrm{~nm}$ for the length of the sensing taper. This length is comparable to etched nanopores in silicon membranes, which have thicknesses from 5 to $20 \mathrm{~nm}^{4,28} \mathrm{SI}$ Figure S7 shows the explicit function of the fit in Figure $3 c$.

Finally, we looked at the noise behavior of the different sized nanocapillaries to calculate the corresponding signal-to-noise ratio and compare it with classical nanopores embedded in a thin $\mathrm{Si}_{3} \mathrm{~N}_{4}$ membrane. Figure $4 \mathrm{a}$ and $\mathrm{b}$ represent typical conductance traces of different sized nanocapillaries at potentials between 0.2 and $0.4 \mathrm{~V}$ with their respective SEM images displayed in the right top corner of the graph. The presented nanocapillaries were also among the ones used for DNA translocation experiments discussed before. Figure $4 \mathrm{c}$ in contrast shows the conductance trace of a nanopore drilled with a TEM into a $\mathrm{Si}_{3} \mathrm{~N}_{4}$ membrane with the respective TEM image. Since SEM requires backscattered electrons and TEM relies on the transmission of electrons, the nanopores are black in the SEM and bright in the TEM image.

To better illustrate the higher noise of the nanopores in silicon nitride, the centered conductance was plotted in a histogram (see Figure $4 d$ ). While the conductance spans over a range of about $0.4 \mathrm{nS}$, for the membrane nanopore it is only $0.2 \mathrm{nS}$ broad for the nanocapillary nanopores. To quantify this noise, the average standard deviation (st. dev.) was calculated by averaging over $10 \mathrm{~ms}$ long conductance traces at $0.1,0.2$, and $0.3 \mathrm{~V}$. This was plotted as a function of the corresponding diameter (see Figure 5a). One can see that the standard deviations of the nanocapillaries are all roughly in the range 0.05 to $0.15 \mathrm{nS}$ (see hollow, blue circles in 

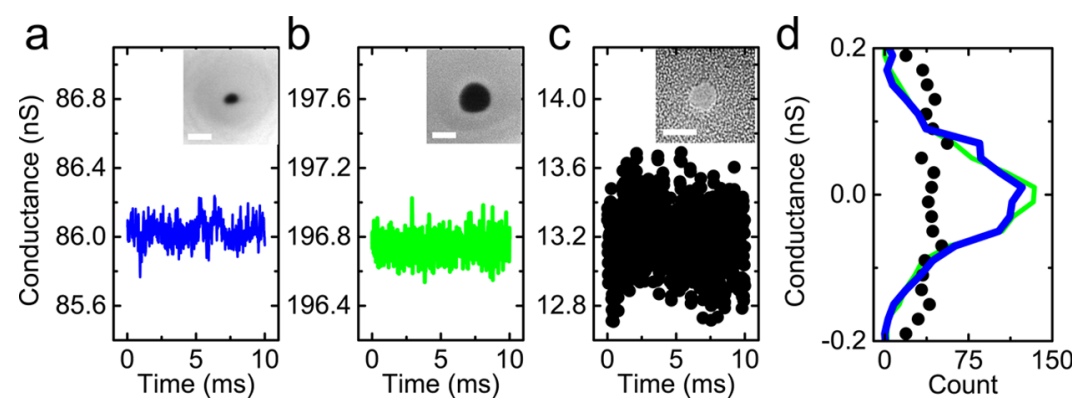

Figure 4. (a) $10 \mathrm{~ms}$ long conductance trace of a $21 \mathrm{~nm}$ diameter nanocapillary. The inset shows the respective shrunken nanocapillary with a $50 \mathrm{~nm}$ wide scale bar. (b) Conductance trace of a $62 \mathrm{~nm}$ wide nanocapillary with the respective SEM image of the nanocapillary with a $50 \mathrm{~nm}$ scale bar. (c) Conductance trace of a $10 \mathrm{~nm}$ wide nanopore in a silicon nitride membrane. The inset shows the TEM image of the nanopore with $10 \mathrm{~nm}$ scale bar. (d) Histogram of the centered conductance traces of the previous nanocapillaries and nanopore. The width of distribution of the nanocapillaries is only $0.2 \mathrm{nS}$, while the histogram from the nanopore spans over $0.4 \mathrm{nS}$.
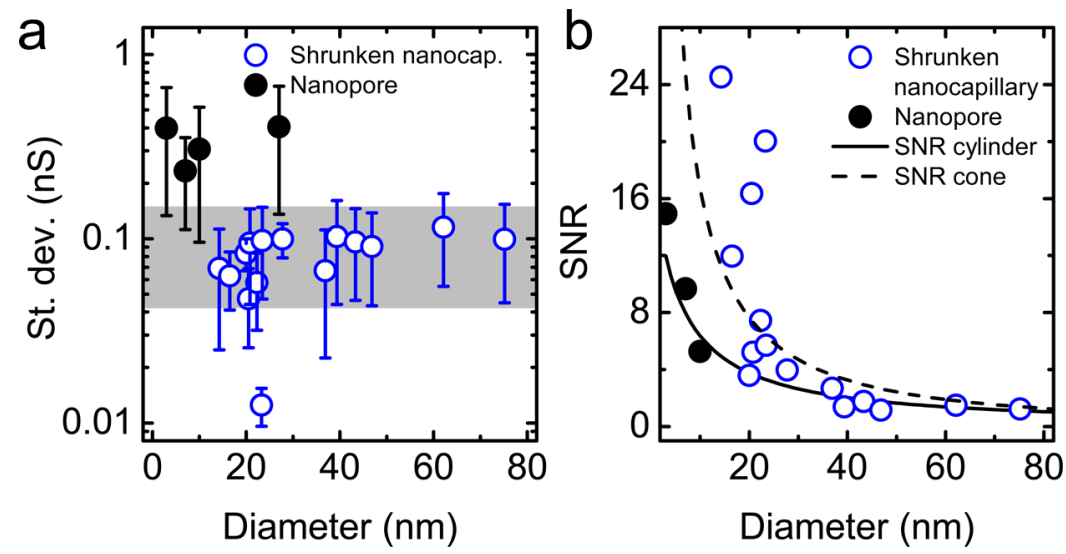

Figure 5. (a) Average standard deviation of $10 \mathrm{~ms}$ long conductance traces at 0.1 to $0.3 \mathrm{~V}$ as a function of the diameter of the nanocapillary (hollow circles) or nanopore (filled circles). The nanocapillaries have approximately 4 times smaller standard deviations than nanopores. The gray band indicates the range of values and spans from 0.04 to $0.15 \mathrm{nS}$ for nanocapillaries, in contrast to nanopores, which are in the range of 0.2 to $0.4 \mathrm{nS}$. (b) Signal-to-noise ratio with respect to the diameter for nanocapillaries and nanopores. The SNR was calculated by dividing the $\Delta G_{\mathrm{dsDNA}}$ value shown in Figure $3 \mathrm{c}$ by the standard deviation from panel (a). The continuous and dashed lines are generated by dividing the model described in eq 3 by the mean standard deviation from panel (a).

Figure $5 \mathrm{a}$ ), in contrast to the cylindrical nanopore in the $20 \mathrm{~nm}$ thick $\mathrm{Si}_{3} \mathrm{~N}_{4}$ membrane, which is more than $0.2 \mathrm{nS}$ (black circles in Figure 5a). A similar noise independence was reported for the length of nanopores in membranes of different thickness by Wanunu et al. ${ }^{22}$ This low-noise characteristic of the ionic conductance was mentioned before, and it is compared with similar cylindrical nanopores in silicon nitride membranes on the same resistive pulse setup. SI Figure S8 shows the conductance power spectral density for all the nanopores and two representative nanocapillaries. It supports our finding with higher $1 / f$ low-frequency and high-frequency noise for the nanopore traces.

Figure $5 b$ depicts the signal-to-noise ratio with respect to the diameter (circles). The hollow circles were calculated by dividing the experimental $\Delta G_{\mathrm{dsDNA}}$ value from Figure $3 c$ with the experimental standard deviation from Figure $5 \mathrm{a}$. The black spheres are data from DNA translocation through classical nanopores in silicon nitride membranes. Typical current traces at 0.1 and $0.2 \mathrm{~V}$ can be seen in SI Figures S9 and S10 for a nanopore with a diameter of $10 \mathrm{~nm}$. As with the nanocapillary data, $\Delta G_{\mathrm{ds} \text { DNA }}$ values were divided by respective standard deviations shown in Figure $5 \mathrm{a}$. The dashed line for the nanocapillary data in Figure $5 \mathrm{~b}$ was calculated by dividing the fit from eq 3 in Figure $3 \mathrm{c}$ by the mean of the standard deviation value of $0.08 \mathrm{nS}$ in Figure 5a. To calculate the SNR for a cylindrical nanopore, the continuous line was calculated by an adapted eq 3 for the geometry of a classical nanopore and dividing it by the mean standard deviation ( $0.3 \mathrm{nS})$ shown in Figure $5 a .{ }^{23}$ Figure $5 \mathrm{~b}$ demonstrates that the SNR for cylindrical nanopores in membranes is better than that for conical nanopores in nanocapillaries at large diameters (starting from $30 \mathrm{~nm}$ ). However, at smaller diameters (below $30 \mathrm{~nm}$ ), the SNR is higher for conical glass nanopores ( 25 for the $14 \mathrm{~nm}$ nanocapillary) than for cylindrical nanopores in silicon nitride (15 for the $3 \mathrm{~nm}$ nanopore). This is important since although conical nanopores have smaller signal amplitudes than cylindrical ones, the higher noise of the latter due to the big membrane allows glass 
TABLE 1. Pulling Parameters with the Pipette Puller P-2000 for the Fabrication of Nanocapillaries with Diameters between 100 and $200 \mathrm{~nm}$

\begin{tabular}{cccccr} 
cycle & heat & filament & velocity & delay & pull \\
\hline 1 & 650 & 0 & 20 & 128 & 0 \\
2 & 650 & 0 & 30 & 128 & 150
\end{tabular}

nanocapillaries to have a better signal-to-noise ratio with diameters below $30 \mathrm{~nm}$.

\section{CONCLUSION}

This work elaborates the effects of SEM-induced shrinking of glass nanocapillaries on the conductance and in particular on the conductance decrease caused by translocating double-stranded DNA. We visualize shrinking in horizontal and vertical dimensions of the nanocapillaries, decrease their size from over $200 \mathrm{~nm}$ to one-tenth of their original sizes, and present successful DNA translocation through 15 nanocapillaries having different diameters. These 15 nanocapillaries span from diameter sizes of 75 to $14 \mathrm{~nm}$, and an equation is developed to model the conductance of conicalshaped nanopores including the unmodified and shrunken nanocapillaries. This model is then utilized for simulation of the blocked conductance caused by the translocation of a single dsDNA molecule. With the help of this model we are able to reproduce the experimental data and estimate the sensing length of our nanocapillaries to around $32 \mathrm{~nm}$. These results show that shrinking glass nanocapillaries puts them very close to state-of-the-art nanopores in membranes regarding their shape and the effect on the amplitude of the conductance decrease for DNA translocation. Finally, the ionic conductance noise behavior of the glass nanopores with various diameters was examined and compared with classical nanopores in membranes. We can demonstrate the signal-to-noise ratio is better for glass nanocapillaries with a diameter below $30 \mathrm{~nm}$ than standard nanopores in silicon nitride membranes. This has important implications on processes such as DNA sequencing, which require very small nanopores but also low-noise characteristics to distinguish the four bases. ${ }^{17}$ One future direction would be, besides coating the nanopore-containing membrane with PDMS, low salt concentration or decreasing the area of the membrane holding the nanopore, to use low-noise materials such as glass. ${ }^{25,29,30}$

\section{METHODS}

The quartz capillaries were purchased with an inner and outer diameter of 0.3 and $0.5 \mathrm{~mm}$, respectively (Hilgenberg, Germany). The capillaries were pulled with a P-2000 laser pipet puller (Sutter, USA). The pulling parameters consisting of a two-line program are listed in Table 1.

This resulted in an elongation of the glass in the first cycle and in the separation of the capillary into two nanocapillaries in the second step. The laser was, on average, activated for $1.2 \pm 0.1 \mathrm{~s}$. The nanocapillaries possessed a mean taper length of $2.1 \mathrm{~mm}$ (see SI Figure S1) and tip diameters between approximately 100 and $200 \mathrm{~nm}$. A detailed description of capillary pulling can be found in a previous publication. ${ }^{26}$

The resulting nanopore at the tip of the nanocapillary was imaged under a field emission scanning electron microscope. The Merlin SEM (Zeiss, Germany) did not necessitate the presence of a conducting layer on the glass nanocapillaries when imaging with the in-lens detector. This allowed determining the diameter of every nanopore before assembling it into the measurement cell. SEM imaging was performed under a working distance between 2 and $4 \mathrm{~mm}$, magnifications between $100 \mathrm{k}$ and $300 \mathrm{k}$, beam currents between 400 and $600 \mathrm{pA}$, and an acceleration voltage of $2 \mathrm{kV}$. Continuous imaging at these conditions from 1 to $5 \mathrm{~min}$ led to shrinking of the nanopore. This was used to decrease the diameter of the orifice at the tip from up to $210 \mathrm{~nm}$ to values down to $14 \mathrm{~nm}$. Previous investigations on silicon nitride and glass nanopores have shown that low acceleration (below $2 \mathrm{kV}$ ) and high beam currents (above $400 \mathrm{pA}$ for glass nanopores) cause faster shrinking than at higher acceleration voltages and lower beam currents. ${ }^{16,31}$ The reason is the heating of the glass by the impinging and decelerated electrons, which melts the glass and causes the glass to shrink in its diameter due to the surface stress. ${ }^{3}$ Higher beam currents increase the number of electrons heating the glass, while low acceleration voltages restrict the penetration depth of the electron to the glass and prevents their premature leaving of the glass. A parallel aligned glass nanopore tip along the axis of the electron beam eases the determination of the nanopore's diameter, prevents charging effects, and enables a uniform shrinking rate. The detailed characterization and explanation of the shrinking behavior of glass nanopores under SEM irradiation were described in a recent paper. ${ }^{16}$

The nanocapillaries were assembled into a PDMS cell, which contained two reservoirs connected only by the nanocapillary. The bottom of the PDMS cell was sealed with a $0.15 \mathrm{~mm}$ thick cover glass (Menzel-Gläser, Germany). The reservoirs were filled with a potassium chloride $(\mathrm{KCl})$ solution of $1 \mathrm{~mol} / \mathrm{L}(\mathrm{M}), 1 \mathrm{mM}$ Tris, and $0.1 \mathrm{mM}$ ethylenediaminetetraacetic acid (EDTA) buffer at $\mathrm{pH}$ 8. The solution was cleared from contaminating particles using an Anotop 25 filter (Whatman, USA). To remove air bubbles inside the nanocapillary after addition of the buffer solution, the PDMS cell was degassed inside a desiccator using a vacuum line. ${ }^{26}$ Oxygen plasma for $5 \mathrm{~min}$ did improve this step by rendering the surface hydrophilic.

An Axopatch 200B current amplifier was used (Axon Instruments, USA) to apply potentials from up to -1 to $+1 \mathrm{~V}$ and measure ionic currents up to $200 \mathrm{nA}$. It has a low-pass Bessel filter at $10 \mathrm{kHz}$, and a PXI-4461 DAQ card (National Instruments, USA) was used to sample the filtered current at a frequency of $100 \mathrm{kHz}$. The silver electrodes were chlorinated $(\mathrm{Ag} / \mathrm{AgCl})$ by applying a dc potential of $2 \mathrm{~V}$ in a $1 \mathrm{M} \mathrm{KCl}$ solution. One $\mathrm{Ag} / \mathrm{AgCl}$ electrode was placed on each side of the nanocapillary (see Figure 1a).

The conductance of the cells was measured by taking a standard current-voltage $(I-V)$ curve. Voltages from a minimum range of -0.3 to $+0.3 \mathrm{~V}$ were applied, and current responses of the cell were plotted to the corresponding potentials to generate the $I-V$ curve. This curve was fitted with a linear function whose slope gave the conductance. Shrunken nanocapillaries with conductance values below $250 \mathrm{nS}$ were chosen for DNA translocation experiments.

Cylindrical nanopores were drilled using a transmission electron microscope (Philips/FEI CM300) in a $20 \mathrm{~nm}$ thick silicon nitride membrane. ${ }^{32}$ The membrane was $500 \mathrm{~nm}^{2}$ big, and the electron beam was operated at a potential of $200 \mathrm{kV}$ and a spot size of 4. After fabrication, the pores were stored in a degassed and filtered 1:1 dd $\mathrm{H}_{2} \mathrm{O} / \mathrm{EtOH}$ solution until use. 
$\lambda$-DNA from New England Biolabs was used for the translocation experiments. The DNA was diluted in the salt solution to a concentration of $0.75 \mathrm{nM}$. This solution was heated to $60^{\circ} \mathrm{C}$ for $5 \mathrm{~min}$ to avoid agglomerated $\lambda$-DNA. Once a positive potential was applied between 0.1 and $0.6 \mathrm{~V}$, characteristic translocation events were observed, which showed decreases in the ionic current with quantized steps. The events were analyzed with the open source program OpenNanopore. ${ }^{33}$

Conflict of Interest: The authors declare no competing financial interest.

Supporting Information Available: This material is available free of charge via the Internet at http://pubs.acs.org.

Acknowledgment. This work was financially supported by the European Research Council (grant no. 259398, ProABEL: Nanopore integrated nanoelectrodes for biomolecular manipulation and design). We specially thank C. Santschi and O. Martin for providing a pipet puller. We also acknowledge P. Granjon, K. Woodruff, K. Liu, and J. Feng for fruitful scientific discussions.

\section{REFERENCES AND NOTES}

1. Li, J.; Stein, D.; McMullan, C.; Branton, D.; Aziz, M. J.; Golovchenko, J. A. Ion-Beam Sculpting at Nanometre Length Scales. Nature 2001, 412, 166-169.

2. Smeets, R. M. M.; Keyser, U. F.; Krapf, D.; Wu, M.-Y.; Dekker, N. H.; Dekker, C. Salt Dependence of Ion Transport and DNA Translocation through Solid-State Nanopores. Nano Lett. 2006, 6, 89-95.

3. Storm, A. J.; Chen, J. H.; Ling, X. S.; Zandbergen, H. W.; Dekker, C. Fabrication of Solid-State Nanopores with Single-Nanometre Precision. Nat. Mater. 2003, 2, 537-540.

4. Kim, M. J.; Wanunu, M.; Bell, D. C.; Meller, A. Rapid Fabrication of Uniformly Sized Nanopores and Nanopore Arrays for Parallel DNA Analysis. Adv. Mater. 2006, 18, 3149-3153.

5. Wu, S.; Park, S. R.; Ling, X. S. Lithography-Free Formation of Nanopores in Plastic Membranes Using Laser Heating. Nano Lett. 2006, 6, 2571-2576.

6. Chansin, G. A. T.; Hong, J.; Dusting, J.; DeMello, A. J.; Albrecht, T.; Edel, J. B. Resizing Metal-Coated Nanopores Using a Scanning Electron Microscope. Small 2011, 7, 2736-2741.

7. Beamish, E.; Kwok, H.; Tabard-Cossa, V.; Godin, M. Precise Control of the Size and Noise of Solid-State Nanopores Using High Electric Fields. Nanotechnology 2012, 23, 405301.

8. Shao, Y.; Mirkin, M. Fast Kinetic Measurements with Nanometer-Sized Pipets. Transfer of Potassium lon from Water into Dichloroethane Facilitated by Dibenzo-18Crown-6. J. Am. Chem. Soc. 1997, 119, 8103-8104.

9. Steinbock, L. J.; Otto, O.; Chimerel, C.; Gornall, J.; Keyser, U. F. Detecting DNA Folding with Nanocapillaries. Nano Lett. 2010, 10, 2493-2497.

10. Steinbock, L. J.; Otto, O.; Skarstam, D. R.; Jahn, S.; Chimerel, C.; Gornall, J. L.; Keyser, U. F. Probing DNA with Micro- and Nanocapillaries and Optical Tweezers. J. Phys.: Condens. Matter 2010, 22, 454113.

11. Otto, O.; Steinbock, L. J.; Wong, D. W.; Gornall, J. L.; Keyser, U. F. Note: Direct Force and lonic-Current Measurements on DNA in a Nanocapillary. Rev. Sci. Instrum. 2011, 82, 086102.

12. Otto, O.; Sturm, S.; Laohakunakorn, N.; Keyser, U. F.; Kroy, K. Rapid Internal Contraction Boosts DNA Friction. Nat. Commun. 2013, 4, 1780-1787.

13. Laohakunakorn, N.; Ghosal, S.; Otto, O.; Misiunas, K.; Keyser, U. F. DNA Interactions in Crowded Nanopores. Nano Lett. 2013, 13, 2798-2802

14. Hernández-Ainsa, S.; Muus, C.; Bell, N. A. W.; Steinbock, L. J.; Thacker, V. V; Keyser, U. F. Lipid-Coated Nanocapillaries for DNA Sensing. Analyst 2012, 16-18.

15. Hernández-Ainsa, S.; Bell, N. A. W.; Thacker, V. V; Göpfrich, K.; Misiunas, K.; Fuentes-Perez, M. E.; Moreno-Herrero, F.; Keyser, U. F. DNA Origami Nanopores for Controlling DNA Translocation. ACS Nano 2013, 7, 6024-6030.
16. Steinbock, L. J.; Steinbock, J. F.; Radenovic, A. Controllable Shrinking and Shaping of Glass Nanocapillaries Under Electron Irradiation. Nano Lett. 2013, 13, 1717-1723.

17. Venta, K.; Shemer, G.; Puster, M.; Rodríguez-Manzo, J. A.; Balan, A.; Rosenstein, J. K.; Shepard, K.; Drndić, M. Differentiation of Short, Single-Stranded DNA Homopolymers in Solid-State Nanopores. ACS Nano 2013, 7, 4629-3466.

18. Li, W.; Bell, N. A. W.; Hernández-Ainsa, S.; Thacker, V. V; Thackray, A. M.; Bujdoso, R.; Keyser, U. F. Single Protein Molecule Detection by Glass Nanopores. ACS Nano 2013, 7, 4129-4134.

19. DeBlois, R. W.; Bean, C. P. Counting and Sizing of Submicron Particles by the Resistive Pulse Technique. Rev. Sci. Instrum. 1970, 41, 909.

20. Saleh, O. A.; Sohn, L. L. Direct Detection of AntibodyAntigen Binding Using an on-Chip Artificial Pore. Proc. Natl. Acad. Sci. U.S.A. 2003, 100, 820-824.

21. Willmott, G. R.; Parry, B. E. T. Resistive Pulse Asymmetry for Nanospheres Passing through Tunable Submicron Pores. J. Appl. Phys. 2011, 109, 094307.

22. Wanunu, M.; Dadosh, T.; Ray, V.; Jin, J.; McReynolds, L.; Drndić, M. Rapid Electronic Detection of Probe-Specific microRNAs Using Thin Nanopore Sensors. Nat. Nanotechnol. 2010, 5, 807-814.

23. Kowalczyk, S. W.; Grosberg, A. Y.; Rabin, Y.; Dekker, C. Modeling the Conductance and DNA Blockade of SolidState Nanopores. Nanotechnology 2011, 22, 315101.

24. Larkin, J.; Henley, R.; Bell, D. C.; Cohen-Karni, T.; Rosenstein, J. K.; Wanunu, M. Slow DNA Transport through Nanopores in Hafnium Oxide Membranes. ACS Nano, in press.

25. Tabard-Cossa, V.; Trivedi, D.; Wiggin, M.; Jetha, N. N.; Marziali, A. Noise Analysis and Reduction in Solid-State Nanopores. Nanotechnology 2007, 18, 305505.

26. Steinbock, L. J.; Keyser, U. F. Nanopore-Based Technology. In Methods in Molecular Biology; Gracheva, M. E., Ed.; Springer Science: Totowa, NJ, 2012; Vol. 870, pp 135-145.

27. Steinbock, L. J.; Lucas, A.; Otto, O.; Keyser, U. F. VoltageDriven Transport of lons and DNA through Nanocapillaries. Electrophoresis 2012, 33, 3480-3487.

28. Li, J.; Gershow, M.; Stein, D.; Brandin, E.; Golovchenko, J. A. DNA Molecules and Configurations in a Solid-State Nanopore Microscope. Nat. Mater. 2003, 2, 611-615.

29. Levis, R. A.; Rae, J. L. The Use of Quartz Patch Pipettes for Low Noise Single Channel Recording. Biophys. J. 1993, 65, 1666-1677.

30. Smeets, R. M. M.; Keyser, U. F.; Dekker, N. H.; Dekker, C. Noise in Solid-State Nanopores. Proc. Natl. Acad. Sci. U.S.A. 2008, 105, 417-421.

31. Prabhu, A. S.; Jubery, T. Z. N.; Freedman, K. J.; Mulero, R.; Dutta, P.; Kim, M. J. Chemically Modified Solid State Nanopores for High Throughput Nanoparticle Separation. J. Phys.: Condens. Matter 2010, 22, 454107.

32. Raillon, C.; Cousin, P.; Traversi, F.; Garcia-Cordero, E.; Hernandez, N.; Radenovic, A. Nanopore Detection of Single Molecule RNAP-DNA Transcription Complex. Nano Lett. 2012, 12, 1157-1164.

33. Raillon, C.; Granjon, P.; Graf, M.; Steinbock, L. J.; Radenovic, A. Fast and Automatic Processing of Multi-Level Events in Nanopore Translocation Experiments. Nanoscale 2012, 4, 4916-4924. 